\title{
Argumentation, confrontation et violence verbale fulgurante
}

Argumentative Processes, Confrontation and Acute Verbal Abuse

\section{Claudine Moïse}

\section{(2) OpenEdition}

12 Journals

Édition électronique

URL : https://journals.openedition.org/aad/1260

DOI : $10.4000 /$ aad. 1260

ISSN : $1565-8961$

Éditeur

Université de Tel-Aviv

Référence électronique

Claudine Moïse, «Argumentation, confrontation et violence verbale fulgurante », Argumentation et Analyse du Discours [En ligne], 8 | 2012, mis en ligne le 15 avril 2012, consulté le 15 février 2023. URL : http://journals.openedition.org/aad/1260 ; DOl : https://doi.org/10.4000/aad.1260

Ce document a été généré automatiquement le 29 septembre 2020.

\section{(c) (i) (3)}

Creative Commons - Attribution - Pas d'Utilisation Commerciale - Pas de Modification 4.0 International - CC BY-NC-ND 4.0

https://creativecommons.org/licenses/by-nc-nd/4.0/ 


\title{
Argumentation, confrontation et violence verbale fulgurante
}

\author{
Argumentative Processes, Confrontation and Acute Verbal Abuse
}

\author{
Claudine Moïse
}

1 Notre groupe de recherche travaille depuis quelques années à définir la violence verbale d'un point de vue linguistique et sociolinguistique (voir pour l'état actuel de nos travaux et de nos publications www.violenceverbale.fr). En croisant nos différents terrains (du milieu scolaire à l'espace public) nous avons montré au fil du temps que la violence verbale fulgurante, au-delà des qualifications péjoratives (Laforest \& Vincent 2004) identifiables (provocation, menace, insulte par exemple), est à considérer comme un processus global qui se manifeste dans des «montées en tension », analysables d'un point de vue interactionnel, et où se jouent rapports de pouvoir, processus de catégorisation, identité du sujet et actions sociales.

2 À partir de nos analyses, nous avons identifié trois types de violence verbale :

3 1. La violence fulgurante est une montée en tension contextualisée qui se décline à travers différentes étapes (incompréhension, négociation, évitement, renchérissement, renforcement...) marquées par des déclencheurs de conflit (matériels ou symboliques), des marqueurs discursifs de rupture (durcisseurs, mots du discours, effets syntaxiques) et des actes de langage dépréciatifs directs (harcèlement, mépris, provocation, déni, insulte...) à visée de domination. Entre autres genres interactionnels concernés, la dispute (dans certaines de ses formes) est probablement l'un de ceux où on peut le mieux l'observer.

4 2. La violence polémique repose sur des actes de langage indirects et implicites, une argumentation et des figures de rhétorique à visée polémique et persuasive. Elle occupe surtout le champ de la politique et de l'humour. Elle repose sur une dimension vexatoire à l'adresse d'un groupe ou d'une personne (ironie, réfutation, arguments ad hominem, etc.). Le pamphlet constitue un bon exemple des genres auxquels on pourrait l'associer. 
5 3. La violence détournée s'actualise dans des interactions consensuelles et coopératives feintes et ambigües (compliment, éloge, flatterie, hyperpolitesse, implicites) à valeur illocutoire contraire et enchâssée à des fins de manipulation et de harcèlement. Pour cette raison, il n'y a pas à proprement parler de type associé à la violence détournée.

6 Ces trois types de violence verbale peuvent s'alimenter les uns les autres à travers différents usages pragmatiques directs ou indirects d'actes menaçants. Nous avons particulièrement analysé dans des publications récentes les mécanismes de la violence verbale fulgurante (Moïse 2006, 2012 entre autres).

7 Dans cet article et dans la lignée d'une réflexion déjà entreprise (Moïse \& SchultzRomain 2010), il s'agit d'analyser les spécificités des modalités argumentatives utilisées dans la violence verbale et, plus particulièrement ici, dans la violence verbale fulgurante au sein d'interactions asymétriques. Quels sont les types d'arguments utilisés ? À quelles fins ? Selon quels effets ? En quoi l'argumentation modifie-t-elle le schéma de la violence verbale fulgurante? En quoi les procédés sont-ils différents de ceux utilisés dans des relations symétriques?

\section{La place de l'argumentation dans la violence verbale fulgurante}

8 Notre travail s'inscrit à la croisée de différentes approches théoriques complémentaires, une conception pragmatique du langage en actes qui fonde les courants sociopragmatiques d'analyse de discours (Laforest \& Moïse 2010), l'analyse conversationnelle (Sacks 1992) et les théories de la politesse (Brown \& Levinson 1987). Nous accordons aussi une large place au contexte, notamment dans le sens où les intentions des locuteurs sont en partie motivées par des places sociales à défendre et une volonté de ne pas perdre la face (Goffman 1959/1973)

9 D'un autre point de vue, il s'agit, pour notre questionnement ici, de nous appuyer sur les théories de l'argumentation qui se sont largement développées (voir pour des synthèses, Plantin 1996, Amossy 2010, 2011 entre autres) depuis les années 90. L'argumentation polémique en jeu dans les interactions, et parfois dans la violence verbale fulgurante, est un type particulier de discours ou d'échanges, basée sur la controverse voire le conflit. Le genre polémique (Amossy \& Burger éds 2011) s'appuie sur des figures rhétoriques caractéristiques (Moïse \& Schultz-Romain 2010) (l'ironie ou la définition polémique par exemple), sur la réfutation lorsqu'elle repose non seulement sur des contre-arguments mais sur des arguments ad hominem, et sur des marques de l'agression (sarcasme, injure), etc. Les procédés argumentatifs polémiques alimentent la controverse dans une visée persuasive mais reposent aussi sur des effets de pathos, élément traditionnel de la rhétorique (Rinn 2008).

$10 \mathrm{Au}$ fil de nos recherches, nous avons élaboré une modélisation de la violence verbale fulgurante (Moïse 2012, entre autres). Sans reprendre dans le détail ce cadre interprétatif, je voudrais en signaler quelques points importants pour mon développement ici-même. Au delà de la construction de la violence verbale dans l'interaction, nous considérons l'émergence de la violence verbale comme indissociable d'éléments contextuels ( les moteurs de la violence verbale »).

11 Les contextes préexistent à l'interaction de même que l'interaction les détermine et en dévoile les éléments pertinents à considérer dans l'analyse, puisque exprimés par les 
locuteurs (l'élaboration des thèmes ou topics, les réparations, les évitements, les renoncements ou les collaborations). Ainsi, la violence verbale est soumise à des déclencheurs, propices à une montée en tension, objets matériels (un événement de rupture comme de l'eau sur un vêtement par exemple), actes de langage (une interpellation) ou valeurs non partagées (le respect, le bruit...). La violence verbale fulgurante est aussi conditionnée par le franchissement de normes sociales qu'elles soient spatiales ou temporelles (un métro bondé, une chaleur étouffante), culturelles ou sociales, qu'elles soient institutionnelles ou familiales (les normes de politesse), par les intentions inassouvies des locuteurs (des objectifs réels ou symboliques à atteindre, une valorisation de soi) et leurs propres émotions (une histoire interactionnelle, des souffrances - Plantin, Doury \& Traverso éds 2000). D'une manière ou d'une autre, les normes ou les objectifs des locuteurs dans l'interaction sont plus ou moins explicités, plus ou moins partagés et donc plus ou moins mouvants, ce qui joue sur l'élaboration de la montée en tension violente.

12 Mais à côté de ces différents éléments contextuels, la montée en tension fulgurante s'articule autour d'actes de langage menaçants (provocation, menace, insulte...), voire les actes de condamnation (accusation, reproche, dénigrement... - Laforest \& Moïse 2010). Un locuteur va réagir à une parole qu'il a ressentie comme violente, parole qui s'appuie sur des gestes, une intonation (rythme, débit, hauteur de la voix), des effets pragmatiques (interpellation, relance, reformulation), des ruptures dans les rituels conversationnels, des malentendus interactionnels (à propos des registres de langue, par exemple), mais aussi des mots du discours (durcisseurs versus adoucisseurs) et des procédés argumentatifs. Les modalités argumentatives sont donc des éléments constitutifs de la violence verbale fulgurante mais largement enchâssés dans d'autres éléments contextuels et interactionnels qui peuvent les orienter, les intensifier ou les empêcher.

13 Je vais donc ici m'attacher à la description d'argumentations constitutives de la violence verbale, sans pour autant décrire la montée en tension dans son ensemble.

\section{Une sociolinguistique impliquée et le choix des données}

14 Notre travail sur la violence verbale a fait l'objet d'une commande du Centre Régional de Documentation Pédagogique en Languedoc Roussillon pour un DVD. Grâce au soutien financier de partenaires publics et privés (SNCF, Transports de l'Agglomération de Montpellier, Ecole Nationale d'Administration Pénitentiaire, Mairie de Montpellier, Education Nationale), ce support a pu voir le jour (Moïse 2012) et se compose de deux volumes, un DVD «comprendre » qui analyse le phénomène de la violence verbale fulgurante et un DVD « agir » qui propose quelques pistes de remédiation.

\subsection{Un corpus d'interactions}

15 Tout ça pour dire que si la sociolinguistique s'appuie sur l'analyse de données authentiques, la recherche sur la violence verbale (notamment la violence verbale dans l'espace public) pose de réelles questions éthiques. Comment capter la violence verbale avec consentement préalable? Comment être prêt quand la tension monte? Pour le DVD qui a requis des saynètes vidéo d'une nécessaire qualité d'images, la captation de 
scènes « réelles » s'est vite avérée impossible : poser la caméra et les techniciens sur un quai de gare et attendre une montée en tension violente ? Demander l'autorisation des locuteurs filmés en caméra cachée pour faire partie d'un DVD? Après réflexion, j'ai opté pour la démarche suivante. Je suis partie de la transcription de mes propres données d'interactions violentes et de celles de collègues sociologue et sociolinguistes ${ }^{1}$ (situations en milieu scolaire, dans l'espace public, dans les transports) pour les proposer à fois à des comédiens et à ceux qui jouaient leur propre rôle (contrôleurs dans les trains, gardien de centre pénitentiaire, personnes à l'accueil de structures...).

Je sais que ce travail de reconstitution ne peut être considéré comme du corpus " authentique »; en effet, les conversations familières sont toujours plus marquées par l'oralité (ruptures, chevauchements, ponctuants, allongements etc.) que les interactions jouées, malgré, dans ce cadre, une grande part d'improvisation des locuteurs et de concertation avec les professionnels rompus à ce type d'interactions institutionnelles. En revanche, ces saynètes constituent, pour la violence verbale à proprement parler, un corpus particulièrement intéressant parce qu'elles rendent compte, par leur nombre, leur qualité et leur diversité, de la complexité et de la variété de la construction interactionnelle et des actes de langage menaçants dans la violence verbale fulgurante.

\section{Les modalités argumentatives dans des interactions asymétriques}

17 Je considère les interactions comme asymétriques quand les statuts institutionnels ou professionnels des locuteurs ne sont pas dans un rapport d'égalité mais d'autorité. Dans une telle situation, le guidage des prises de parole n'est pas libre mais induit par le détenteur de pouvoir et les routines ritualisées sont marquées par une distance discursive. "Les relations asymétriques sont révélatrices de règles, de valeurs, de normes de comportement et forcent le recours des interlocuteurs à diverses stratégies d'adaptation » (Laforest \& Vincent $2006: 8$ ).

Il s'agit donc ici, à partir de saynètes de violence verbale fulgurante, de voir quelles sont les «stratégies d'adaptation » argumentatives utilisées, et de quelles façons, dans des montées en tension asymétriques. Dans les situations que je présente, un des interlocuteurs est pris en défaut par le représentant de l'institution (SNCF, Agent de Surveillance de la Voie Publique - ASVP -, Service Pénitentiaire d'Insertion et de Probation - SPIP -) et tente d'une façon ou d'une autre de s'expliquer pour déjouer la sanction possible.

\subsection{L'argumentation par le lien causal}

\footnotetext{
Une passagère (P2) a pris le train sans avoir eu le temps de composter son billet. La règle veut que, dans ce cas, tout voyageur se présente au contrôleur avant le contrôle.

((Un train roule une jeune femme (P2) lit et écoute de la musique avec son baladeur. Une agente SNCF arrive pour le contrôle des billets. Bruit régulier.))

1. Contrôleuse (C) : < messieurs dames bonjour vérification des titres de transport s'il vous plaît $>(($ au loin hors champ $))<$ messieurs dames bonjour vérification des titres de transport bonjour madame > ((elle s'approche, une passagère (P2) ne l'entend pas, elle contrôle le billet d'une autre passagère (P1) qui le lui tend avec le sourire))
} 
2. Passagère $1:$ bonjour

3. $C:<$ je vous remercie $>(($ elle composte le billet $)) \underline{\text { merci bien }}$

4. $\mathrm{P} 1:<$ merci $>(($ la contrôleuse lui rend son billet $))$

5. $\mathrm{C}:$ mademoiselle bonjour vérification des titres de transport

6. P2 : ((elle reste plongée dans la lecture et la musique, elle tend son billet sans regarder la contrôleuse))

7. $\mathrm{C}:$ < vous n'avez pas composté votre billet $\operatorname{mad}(\mathrm{e})$ moiselle > ((P2 ne réagit pas $))$

vous n'avez pas composté votre

8. $\mathrm{P} 2:<$ comment > ((elle enlève un de ses écouteurs et tourne la tête vers la contrôleuse) $)$

9. $C$ : vous n'avez pas composté votre billet +

10. $P 2$ : bé en fait $E: j(e)$ suis rentrée $E: j$ 'ai failli rater le train $j(e)$ je suis arrivée en CoUrant $\mathrm{j}(\mathrm{e})$ suis rentrée dans $\mathrm{l}(\mathrm{e})$ train je me suis assise à ma place (il) y avait tellement $d(e)$ monde du coup je me suis assise et $E$ :

11. $\mathrm{C}: \underline{\text { alors }}$

12. $\mathrm{P} 2$ : et voilà du coup $\mathrm{j}(\mathrm{e})$ (n') ai pas eu $\mathrm{l}(\mathrm{e})$ temps quoi en fait

13. $C$ : quand c'est comme ça il vous appartient de venir sur le quai nous le signaler + hein quand vous n' avez pas le temps de composter votre billet

14. P2 : ben j(e) vous ai pas vu madame j(e) vous ai pas vue avec le monde qu'i(l) (y) avait là là c'était la panique sur le quai là avec les vacances

15. C. : et pourtant on est toujours sur le quai en voiture numéro trois + il vous appartient de venir dire que vous n'avez pas eu le temps de composter le billet hein (2s) donc un billet non composté c'est dix euros mademoiselle

16. P2 : non mais attendez j(e) (n')y crois pas vous allez quand même pas me faire payer pour ça non mais j'hallucine attendez j'ai payé mon billet déjà j(e) l'ai pris à l'avance + et en plus vous allez me faire payer dix euros

17. C : alors vous avez payé votre billet il vous appartient de le composter vous ne l'avez pas composté ça va faire dix euros

18. $\mathrm{P} 2:<\mathrm{xx}$ j'hallucine la la non non mais $((\underline{\mathrm{pff} f \mathrm{ff}}))>(($ elle s'énerve $))$

19. $C$ : vous payez comment s'il vous plaît

20. P2: $\underline{((p f f p f f f f)})$ i(l) manquait qu(e) ça non mais quelle journée de MERde vraiment (2s) non mais oui ((pff)) $\mathrm{j}(\mathrm{e})$ sais pas je vais regarder ((elle fouille dans son sac à main))

Dans cette saynète où la tension est latente (la passagère, au tout début, tend son billet à l'agente SNCF sans la regarder et sans aucun signe de politesse) et où donc la provocation par une forme d'irrespect du travail de l'autre est significative, le jeu argumentatif va, d'une certaine façon, servir la contrôleuse au détriment de la passagère.

L'agente de la SNCF doit se faire respecter et appliquer le règlement, la passagère, de son côté, a sans doute pour objectif d'être (enfin) tranquille. Au tout début, la contrôleuse commence par une demande polie avec mots d'adresse («mademoiselle bonjour » (tour 5) mais qui reste sans réponse. Et donc l'interaction suivante (tour 7) se construit comme un reproche (action déclarée qui ne correspond pas aux attentes d'un interlocuteur, voir Laforest 2002) (7. C: < vous n'avez pas composté votre billet $\operatorname{mad}(\mathrm{e})$ moiselle $>$ ) qu'elle doit formuler trois fois (tours 7-9).

21 Pour tenter de se défendre, la passagère P2 (tours 10-12) va user, dans une visée argumentative, d'un procédé des plus simples, le lien causal explicatif (Plantin 1996: 41 ) ; le manque de temps (lien explicatif causal marqué par le « et voilà » conclusif) l'a empêchée de composter son billet (événement) :

10. $P 2$ : bé en fait $E: j(e)$ suis rentrée $E: j$ 'ai failli rater le train $j(e)$ je suis arrivée en CoUrant j'(e) suis rentrée dans $1(\mathrm{e})$ train je me suis assise à ma place (il) y avait tellement $\mathrm{d}(\mathrm{e})$ monde du coup je me suis assise et $\mathrm{E}$ :

11. $\mathrm{C}: \underline{\text { alors }}$ 
12. P2 : et voilà du coup j'ai pas eu $1(\mathrm{e})$ temps quoi en fait

L'agente de la SNCF n'a même pas besoin de réfuter l'argumentation causale (« vous auriez pu prendre le temps de composter » ou « vous auriez pu prévoir » par exemple), ce qui aurait sans doute amené une discussion-polémique autour de la possibilité de l'action ou pas (composter son billet). Elle va argumenter, avec une certaine force, par une concession. Bien que le billet n'ait pas été composté, la passagère effectivement avait droit à l'erreur; elle devait toutefoissignaler son erreur. L'agente n'a donc pas à prouver que la justification est acceptable, elle est d'accord sur ce fait. Par là même, elle va déplacer la possible opposition.

La forme de culpabilisation et de leçon donnée à la passagère marquée par le " hein » (13. C : quand c'est comme ça + il vous appartient de nous le signaler hein), entraîne alors un renchérissement par justification de la part de la passagère, qui reste hésitante mais qui va contre-argumenter encore une fois par une explication causale, le monde et la panique l'ont empêchée de voir l'agente de la SNCF (tour 14) (14. P2 : ben j(e)) vous ai pas vu madame $\mathrm{j}(\mathrm{e})$ vous ai pas vue avec le monde qu'i(l) (y) avait là là c'était la panique sur le quai là avec les vacances).

Il s'agit dans cet échange d'une argumentation de la part de P2 par «le poids des choses » à laquelle peut s'opposer une argumentation « volontariste ». L'argumentation volontariste, qui vise à nier un certain déterminisme - déterminisme du type « je n'y peux rien » utilisé par P2 -, est avancée par la contrôleuse, non pas sur le premier argument qu'elle concède (ne pas composter son billet) mais sur le second (ne pas l'avoir vue - tour 15). Elle le réfute en montrant que la passagère aurait pu être plus vigilante et donc à la hauteur de la situation (15. C : et pourtant on est toujours sur le quai en voiture numéro trois il vous appartient de venir dire que vous n'avez pas eu le temps de composter le billet hein (2s) donc un billet non composté c'est dix euros mademoiselle). «L'argumentation volontariste permet de rejeter l'argumentation par le poids des choses et d'ouvrir sur la figure classique de l'homme de la situation " (Plantin 1996: 44). La conclusion (marquée par le "donc») de la déduction est impitoyable (15. C : donc un billet non composté c'est dix euros mademoiselle »).

On peut penser que si la passagère n'avait pas empiété sur le territoire de l'agent donc sur la reconnaissance de son rôle institutionnel, les interactions auraient pu s'enchaîner avec plus de bienveillance. La dernière réaction, énervée, de la passagère est à la hauteur de son propre échec argumentatif dans la mesure où, de son côté, elle ne s'est pas sentie entendue (tours 16-20). Malgré tout, parce que l'argument de la contrôleuse est martelé sans concession, que c'est quasiment un argument de droit voire définitoire, auquel elle ne se voit opposer aucun contre-argument (17. C: alors vous avez payé votre billet il vous appartient de le composter vous ne l'avez pas composté ça va faire dix euros), la passagère va retourner vers elle la tension par un juron (donc adressé à soi-même) (20. P2 : quelle journée de MERde).

Au terme de cette première analyse, on peut dire que la montée en tension est bien repérable par l'expression d'une certaine exaspération (tours 16 et 18). Une bascule serait alors possible dans une plus grande violence verbale, fulgurante et vive. La passagère renonce à transgresser davantage le règlement, ce qui aurait pu l'amener à s'emporter davantage et à se mettre dans une situation délicate. 


\subsection{L'argumentation par la réfutation ironique}

Un homme, condamné pour conduite en état d'ivresse, se rend à sa convocation au Service Pénitentiaire d'Insertion et de Probation (SPIP) pour l'entretien de mise en place des mesures judiciaires.

((La personne (L1) arrive à pied au SPIP, regarde la plaque du Ministère de la Justice et va entrer. Il essaie d'ouvrir la porte qui reste close, il appuie sur un bouton puis sur une sonnette. La porte s'ouvre et il se retrouve dans un sas. Il est inquiet et énervé. Il appuie sur un bouton, secoue la porte, s'énerve. La secrétaire (S) ouvre, il entre.))

((Après l'accueil de la secrétaire, il part vers la salle d'attente, remet sa convocation dans sa poche arrière de pantalon, s'assoit en position relâchée. La conseillère (C) arrive.))

[...]

1. C. : bonjour (1s) monsieur R.

2. $\mathrm{L} 1$ : bonjour madame $\mathrm{U}$.

3. C. <suivez moi monsieur> ((il se lèvent et se serrent la main))

4. L1 : bonjour

((Dans le bureau de Mme U.))

5. C. : installez-vous s'il vous plaît monsieur R. ((ils s'assoient face à face $))+$ c'est la première fois que : vous venez dans nos services $\mathrm{E}$ :

6. L1 : ouais

7. C.: d'accord (1s) E: lors de cet entretien je vais vous expliquer E: la mesure judiciaire hein dont vous faites l'objet

8. L1 : ouais très bien si on pouvait faire vite parce que j'ai un ami qui m'attend dehors ((montre la fenêtre)) comme vous $1(e)$ savez $\mathrm{j}(\mathrm{e})(\mathrm{n}$ ') ai plus mon permis $\mathrm{d}(\mathrm{e})$ conduire

9. C. : j'ai un $\mathrm{C}<+$

10. L1 : et donc je suis tributaire des autres

11. C. : j'ai un certain nombre d'informations à vous délivrer hein en l'occurrence vous avez été condamné donc par le tribunal correctionnel <+

12. L1 : à six mois de retrait de permis je le sais si on pouvait avancer merci <+

13. C. : vous avez été condamné à une peine de sursis avec mise à l'épreuve $\mathrm{E}$ : une mise à l'épreuve sur un délai de dix-huit mois pour des faits de conduite sous l'emprise de l'état alcoolique mm : c'est une peine qui est sursis

14. L1 : oui c c'est c'est c'est c'est une peine $\mathrm{E}:$ moi je trouve injuste quand même hein je tiens à vous $\mathrm{l}(\mathrm{e})$ dire $\mathrm{xx}$ à vous

15. C. : on (n')est pas là $\mathrm{E}:$ on (n')est pas du tout là pour juger ou refaire le jugement $<+$

16. L1 : ouais ah ouais de tout(es) façons le sursis < ça compte pas hein c'est ça qui est bien > ((débit accéléré) $)$

17. C. : c'est à dire qu'il faudra que vous respectiez un certain nombre d'obligations liées à la mesure judiciaire <+

18. L1 : ouais notamment celle de venir ici aujourd'hui cela est fait

19. C. : des o des obligations générales et des obligations particulières (1s) d'accord

20. L1: je sais oui

21. C.: en cas de non respect effectivement un rapport d'incident pourra être adressé au magistrat qui pourra décider d'une éventuelle révocation et transformation en $\mathrm{d}(\mathrm{e})$ la peine ferme < concernant les obligations générales donc vous devez vous présenter aux convocations > ((il se recule et s'enfonce dans son fauteuil, croise les doigts))

22. $\mathrm{L} 1$ : c'est fait ça y est $\mathrm{j}(\mathrm{e})$ suis là ((il sourit avec ironie))

23. C. : et ce pendant un délai de dix-huit mois

24. L1 : PARDON

25. C. : c'est à dire que tous les mois vous devrez vous présenter au service

26. L1 : < bé c'est à dire que pendant dix-huit mois : je vais devoir venir tous les mois ici venir vous voir demander à quelqu'un de m'accompagner pendant dix-huit mois com(me) vous le savez j'ai pas l(e) permis grâce à vous hein voilà donc (il) y a 
quelqu'un qui pendant dix-huit mois va venir m'emmener une fois par mois pour venir vous voir pour vous dire pour vous dire quoi que je vais bien c'est ça > ((il fait beaucoup de gestes avec les mains et les bras))

27. C : alors moi je n'ai rien à voir dans la condamnation hein $<+$

28. $\mathrm{L} 1$ : X c'est ça je vais venir vous dire que je vais bien c'est ça (que) vous voulez savoir (1s) eh bien je vais bien

29. C. : monsieur

30. L1 : ben voilà je vous enverrai un p(e)tit mail on peut faire ça par mail peut-être

31. C. : on ne peut pas faire ça par mail monsieur R. <+

32. $\mathrm{L} 1:$ mais vous non

33. C. : puisqu'i(l) y a une condamnation judiciaire pour des faits délictuels <+

34. L1 : c'est ça X $\mathrm{j}(\mathrm{e})$ vais perdre une journée de travail par mois pour faire plaisir à la justice quoi c'est ça

35. C. : vous avez (1s) commis des faits qui sont répréhensibles vous avez été condamné <+

36. L1 : des faits oui oui j'ai commis des faits j'ai jamais eu d'accident de voiture j(e) suis pas un criminel < j(ai) jamais eu un $\mathrm{PV}>$ ((il compte sur ses doigts pour énumérer $)$ ) et et là vous m'accusez de de comme un criminel quoi j'ai l'impression ((s'arrête de parler et recule pour s'asseoir au fond de son fauteuil))

37. C. : je ne juge pas de votre état j'essaie de mettre en place $\mathrm{E}:$ la mesure judiciaire donc pendant (2s) dix-huit mois vous vous présenterez tous les mois ici

38. L1 : bien voilà < d'accord > ((il frappe sur le bureau $)$ ) tous les mois j(e) m(e) présente $<+$

39. C.: vous avez également (1s) vous avez (1s) également une obligation particulière liée à cette mesure

40. L1 : en plus

41. C. : oui vous avez une obligation de soins

42. L1 : de soins c'est-à-dire que $\mathrm{j}(\mathrm{e})$ suis malade aussi main(te)nant + vous allez pas m'enfermer à l'asile aussi tant que vous y êtes ((très énervé))

43. C. : vous devrez mettre en $p<+$

44. L1 : vous avez rien trouvé de mieux pour pour aider les gens (1s) que les mettre dans l(e) trou dans l'entonnoir c'est tout ce (que) vous avez trouvé < ah là BRAVO je vous félicite hein > ((ironique et en colère)) ((il applaudit))

45. C. : vous devrez vous devrez

46. L1 : bravo bravo ((il applaudit)) + justice de merde

Monsieur R. vient pour un entretien de mise en place de mesures judiciaires suite à une conduite en état d'ivresse. Déjà, avant de rencontrer la conseillère, il paraissait nerveux, inquiet, sans comprendre pourquoi il était convoqué.

Même s'ils sont respectés, les rituels conversationnels initiés par la conseillère, au-delà des préservations des faces ou d'attention à l'autre, servent, par leur marquage, de mise à distance et de rappel de l'asymétrie des places (1. C : bonjour (1s) monsieur R. / 2. L1 : bonjour madame U. / 3. C. <suivez moi monsieur> ((il se lèvent et se serrent la main)) / 5. C : installez-vous s'il vous plaît monsieur R). Tout au long de l'échange, la conseillère n'a de cesse de poser ses propres règles interactionnelles et institutionnelles en exposant à son interlocuteur la procédure à suivre. Il est frappant de voir à quel point elle refuse d'entrer dans l'interaction et poursuit son but, faire passer ses explications. Elle ne manifeste ni coopération, ni négociation, ni régulation :

7. C.: d'accord (1s) E: lors de cet entretien je vais vous expliquer E : la mesure judiciaire hein dont vous faites l'objet

8. L1: ouais très bien si on pouvait faire vite parce que j'ai un ami qui m'attend dehors ((montre la fenêtre)) comme vous l(e) savez j(e) (n') ai plus mon permis d(e) conduire

9. C. : j'ai un $\mathrm{c}<+$ 
10. L1 : et donc je suis tributaire des autres

11. C. : j'ai un certain nombre d'informations à vous délivrer hein en l'occurrence vous avez été condamné donc par le tribunal correctionnel <+

Face à un tel fonctionnement interactionnel (qui est sans doute exigé par l'institution), le locuteur (L1) n'a aucune prise. Le but est qu'il se plie à l'injonction de suivi. Diverses stratégies sont alors possibles, il peut coopérer, écouter et acquiescer ; il peut exploser et proférer des actes menaçants directs (provocation, menace, insulte); il choisit ici d'argumenter de façon indirecte, par la réfutation et une certaine ironie (voir sur le sens de l'ironie Perrin 1996), façon de manifester sa désapprobation et sa colère.

L1 commence par manifester de l'insolence, c'est-à-dire qu'il va inverser le rapport de places, asymétrique, pour s'arroger le temps de son interaction une place haute. Au tour 12, il disqualifie l'information donnée par la conseillère en signifiant qu'il est au courant « je le sais », et en employant une injonction «si on pouvait avancer », qui lui donne l'initiative de l'interaction, et un « merci » ironique (12. L1 : à six mois de retrait de permis je le sais si on pouvait avancer merci $<+$ ).

1 Tandis que la conseillère poursuit son propos sans tenir compte de cette intervention, L1 exprime encore une réfutation de la peine et donc de la mise à l'épreuve, pour la raison morale qu'il la considère comme injuste, ce qu'il va tenter par la suite d'expliquer (14. L1 : oui c c'est c'est c'est c'est une peine $\mathrm{E}$ : moi je trouve injuste quand même hein je tiens à vous $1(\mathrm{e})$ dire xx à vous). Argument balayé par la conseillère au tour suivant comme étant inapproprié au contexte institutionnel (15. C. : on (n')est pas là $\mathrm{E}$ : on (n')est pas du tout là pour juger ou refaire le jugement).

32 Quand il comprend qu'il doit se présenter mensuellement, et pendant dix-huit mois, dans ce service, il le refuse mais, encore une fois, indirectement en montrant l'absurdité de la situation : il faudrait mobiliser quelqu'un, puisqu'il n'a plus le permis, et pour simplement venir signifier qu'il va bien. Tandis que la conseillère, comme précédemment, ne prend pas en compte cette interaction, en se réfugiant à nouveau derrière des prérogatives institutionnelles (25. C : alors moi je n'ai rien à voir dans la condamnation hein $<+)$, L1 va filer son refus (venir à une convocation mensuelle) avec ironie, qui minimise et disqualifie la condamnation, voire la dénie (30. L1 : ben voilà je vous enverrai un p(e)tit mail on peut faire ça par mail peut-être). La conseillère, de sa place, va encore réfuter catégoriquement cette proposition (31. C. : on ne peut pas faire ça par mail monsieur R.).

la conseillère prononce le mot " condamné » (tour 35) (35. C.: vous avez (1s) commis des faits qui sont répréhensibles vous avez été condamné <+), l'argumentation par réfutation prend un autre tour et s'apparente clairement à du déni (refus d'accepter l'événement dans sa réalité) (36. L1 : des faits oui oui j'ai commis des faits j'ai jamais eu d'accident de voiture $j(e)$ suis pas un criminel $<j(a i)$ jamais eu un PV > ((il compte sur ses doigts pour énumérer)) et et là vous m'accusez de de comme un criminel quoi j'ai l'impression ((s'arrête de parler et recule pour s'asseoir au fond de son fauteuil)). L1 refuse de considérer la conduite en état d'ivresse comme condamnable, et récuse l'accusation, comme le montre le chevauchement et la concession (voir pour la concession Vincent \& Heisler 1999) (36. L1 : des faits oui oui j'ai commis des faits j'ai jamais eu d'accident de voiture $\mathrm{j}(\mathrm{e})$ suis pas un criminel), avec toujours le même procédé d'évitement de la part de la conseillère (37. C. : je ne juge pas de votre état).

Quand, au tour 41, la conseillère renchérit sur l'obligation de soins (41. C.: oui vous avez une obligation de soins), le déni prend toute sa force. $\mathrm{L} 1$ refuse de se considérer 
comme alcoolique avec la nécessité d'être pris en charge pour des soins adaptés (42. L1 : de soins c'est-à-dire que j(e) suis malade aussi main(te)nant + vous allez pas m'enfermer à l'asile aussi tant que vous y êtes ((très énervé)). Déni qui sera marqué au tour 44 par une question et un acte de félicitation ironiques avec encore cette fois-ci retournement des places asymétriques dans un effet d'insolence (44. L1: vous avez rien trouvé de mieux pour pour aider les gens (1s) que les mettre dans l(e) trou dans l'entonnoir c'est tout ce (que) vous avez trouvé < ah là BRAVO je vous félicite hein > ((ironique et en colère)) ((il applaudit)). D'une certaine façon, face aux règlements, lois et contraintes énoncés par C., L1 oppose une demande d'humanité et de soutien.

Parce qu'il n'y a pas eu de réel échange interactionnel, L1 utilise en dernier recours, comme bien souvent dans la violence verbale fulgurante, une insulte indirecte (44. L1 : bravo bravo ((il applaudit)) + justice de merde), proférée à l'égard d'un tiers, la Justice, et non pas vis-à-vis de $C$., ce qui, dans une visée abstraite, désincarnée et globalisante, en amoindrit la menace et la portée, dans ce cadre asymétrique.

Ainsi, face à un cadre interactionnel et une progression séquentielle imposés par la conseillère, L1 tente d'inverser le rapport asymétrique et, sans oser aller jusqu'à l'affrontement direct, use de procédés argumentatifs indirects de réfutation ironique. Là encore, on peut dire sans doute que les procédés argumentatifs, s'ils permettent une confrontation, évitent d'entrer dans une violence verbale fulgurante caractérisée.

\section{3 : L'argumentation par la concession}

Un passager, qui a besoin d'une prise de courant pour brancher son ordinateur, s'installe en première classe alors qu'il a un billet de seconde.

((Un passager ( $P$ ) dans un wagon se sert de son ordinateur)) ((Bruit du train qui roule et voix off: "Mesdames et messieurs bonjour vous avez pris place à bord du TGV 6216 à destination de Paris »)) ((une agente de la SNCF se présente (C)))

1. C : monsieur bonjour vérification des titres de transport s'il vous plaît

2. P : ((n'arrête pas de taper sur son clavier et la regarde juste pour lui répondre)) oui j'ai déjà été contrôlé

3. $C$ : bé écoutez ce sera la seconde fois vous avez votre billet s'il vous plaît

4. P : ou : oui pff : ((il souffle, prend le billet et le donne, croise les bras, mécontent))

5. C : merci (3s) ((elle tourne le billet)) vous avez un billet de seconde vous êtes en première là hein

6. $P$ : non mais je sais j'en ai juste pour cinq minutes c'est parce que j(e)vais recharger mon portable (il) y a plus de batterie donc je reviens dans cinq j'y $\mathrm{r}(\mathrm{e})$ tourne dans cinq minutes c'est bon

7. $C$ : oui mais vous étiez déjà là tout à l'heure quand je suis passée vous êtes encor(e) là + alors écoutez moi je vais vous : régulariser hein

8. $\mathrm{P}$ : vous allez $\mathrm{m}(\mathrm{e})$ verbaliser pour ça

9. C : je vais pas vous verbaliser monsieur je vais vous régulariser c'(est) à dire que je vais ramener votre billet à un tarif première (1s) puisque vous avez un billet de seconde

10. $P$ : $m($ ais) attendez j'en ai pour cinq minutes / je recharge je retourn(e) là-bas (1s) < qu'est-ce (que) ça peut vous fair (e) > ((le ton monte) )

11. $C$ : ça $m(e)$ fait rien mais je suis là pour ça

12. $\mathrm{P}$ : mais vot(re) collègue est passé $\mathrm{t}$ (out) à l'heure il (n')a rien dit

13. $C$ : oui mais mon collègue c'est mon collègue et moi c'est moi

14. $\mathrm{P}:$ ah vous êtes un robot vous

15. $\mathrm{C}$ : je vais vous faire le calcul monsieur hein 
16. $\mathrm{P}$ : ah c'est ça ((secoue la tête, en colère)) ah déjà qu'on (n) a pas $\mathrm{d}(\mathrm{e})$ prise de l'aut(re) côté (1s) y a pas d(e) wifi alors on peut mêm(e) pas rêver hein la SNCF c'est médiéval (1s) et en plus là comm(e) ça $\mathrm{E}:$ pas $\mathrm{d}(\mathrm{e})$ cœur quoi rien

17. $C$ : alors vous avez un billet Montpellier Paris : et un surclassement en première ça va vous faire quarante euros monsieur

18. P : QUARANTE EUROS

19. C : quarante

Au début de l'échange, le passager admet qu'il ne devrait pas être là mais demande une certaine indulgence et que cinq minutes lui soient accordées (6. P : non mais je sais j'en ai juste pour cinq minutes c'est parce que j(e)vais recharger mon portable (il) y a plus de batterie donc je reviens dans cinq j'y r(e)tourne dans cinq minutes c'est bon). Sans doute, la négociation est-elle mal engagée à cause de la déconsidération qu'il manifeste à l'égard de l'agente, dès les premiers tours (tours 2 et 4 ), quand elle lui demande son billet. La concession (Vincent \& Heisler 1999) «non mais je sais", suivie d'une explication/justification n'est pas entendue, avant tout parce qu'elle ne semble s'appuyer ni sur un principe de véracité (7. C : oui mais vous étiez déjà là tout à l'heure quand je suis passée vous êtes encor(e) là + alors écoutez), ni de respect (le « c'est bon ", au tour 6, marque une tentative de retournement de l'asymétrie et donc d'une certaine forme de déconsidération pour la fonction). Toute négociation, parce qu'elle repose sur une préservation de l'image et des intérêts de chacun des acteurs (pouvoir recharger le portable et faire respecter le règlement sans frustration de part et d'autre), suppose confiance et respect de part et d'autre. Comme la contrôleuse refuse la tentative de négociation proposée par le passager, elle avance un contre-argument définitoire à propos du verbe « régulariser » (9. C : je vais pas vous verbaliser monsieur je vais vous régulariser $c^{\prime}$ (est) à dire que je vais ramener votre billet à un tarif première (1s) puisque vous avez un billet de seconde). Ce genre de formule utilise la structure de la définition, "non pour fournir le sens d'un mot, mais pour mettre en vedette certains aspects d'une réalité qui risquerait de rester à l'arrière-plan » (Perelman \& OlbrechtsTyteca 1988 : 233) et qui clôt l'argumentation par sa précision.

Le passager, face à l'échec de la concession qu'il a proposée et qu'il réitère (tour 10), va basculer ici, contrairement aux précédentes situations, dans la l'attaque ad personam (Amossy 2010 : 143), et une condamnation du faire puis de l'être, avec le « vous » (10. P : qu'est-ce (que) ça peut vous fair(e)) / 14. P : ah vous êtes un robot vous). La série d'arguments avancée juste après arrive trop tard; elle est de l'ordre du reproche et ne peut être ressentie que comme une condamnation de l'être, en l'occurrence de la SNCF incarnée par la contrôleuse (16. P : ah c'est ça ((secoue la tête, en colère)) ah déjà qu'on (n) a pas $d(e)$ prise de l'aut(re) côté (1s) y a pas $d(e)$ wifi alors on peut mêm(e) pas rêver hein la SNCF c'est médiéval (1s) et en plus là comm(e) ça $\mathrm{E}:$ pas $\mathrm{d}(\mathrm{e})$ cœur quoi rien).

\subsection{L'argumentation narrative et l'effet de pathos}

La mise en récit est une forme très efficace de l'argumentation et donc de la persuasion. Storytelling, elle est utilisée dans les discours politiques notamment : comment raconter des histoires pour émouvoir le public et être efficace?

((Dans la rue, deux agents de surveillance (AS) de la voie publique, font leur travail. L'un deux remplit une contravention quand la propriétaire $(P)$ de la voiture concernée arrive tout sourire))

1. P : c'est bon monsieur j'y vais j'y vais

2. AS : bonjour madame $<+$ 
3. $P$ : j'ai fini regardez je je je m'en allais là

4. AS : $\mathrm{j}(\mathrm{e})$ suis désolé mon PV est fini madame hein

5. P : bé oui mais en même temps j(e) m'en vais enfin : ça c'est pas grave vous :

l'avez pas encore mis ça fait rien

6. AS : ah oui non mais : il est fini donc $\mathrm{j}(\mathrm{e})$ peux plus je peux plus le reprendre quoi $\mathrm{j}(\mathrm{e})$ suis obligé de vous le laisser hein

7. $\mathrm{P}$ : bé là $\mathrm{j}(\mathrm{e})$ comprends mais j'étais juste partie acheter une $\mathrm{p}(\mathrm{e})$ tite bricole pour ma fille parce que (1s) c'est son anniversaire demain et puis comme son père nous a laissées $(2 \mathrm{~s}) \mathrm{j}(\mathrm{e})$ voulais qu'elle (ne) se sente pas : enfin vous voyez $\mathrm{j}(\mathrm{e})$ voulais faire un geste quoi

8. AS : j(e) comprends bien madame mais bon nous une fois qu'on a fini le PV on est obligés de $1(\mathrm{e})$ poser

9. $P$ : bé voui mais : mais enfin là c'est bon $j(e)$ m'en vais regardez m'enfin pouvez pas faire pouvez pas faire que(l)que chose parce que <+

10. AS : je peux je (ne) peux rien faire madame par contre vous avez quarante cinq jours pour le payer $<+$

11. $P$ : j'ai j(e) (n') ai pas beaucoup non mais (en)fin que ce soit quarante cinq jours ou aujourd'hui c'est pareil de toutes façons j(e) (n')ai pas d'argent j(e) (n')ai pas d'argent hein hein avec le le mon mari qui nous $a$ : laissé tomber là bon là moi $j(e)$ fais $c(e)$ que $j(e)$ peux j'achète une bricole à ma fille pas chère pour marquer $1(e)$ coup maintenant si vous me mettez un PV enfin c'est c(e) (n') est pas la peine ça (ne) sert à rien je (ne) pourrai pas payer de toute manière

12. AS : ah oui madame mais moi je (ne) peux pas repartir avec donc j(e) suis obligé de vous l(e) laisser

13. $\mathrm{P}$ : mais allez mais c'est débile votre fonctionnement là (en)fin ça (ne) sert à rien c'est con quoi parce que de toutes façons $\mathrm{j}(\mathrm{e})$ (ne) peux pas $\mathrm{l}(\mathrm{e})$ payer ((il pose le PV sur le pare-brise) $\mathrm{j}(\mathrm{e})(\mathrm{ne})$ peux pas $\mathrm{j}(\mathrm{e}) \mathrm{j}(\mathrm{e})$ (ne) peux pas c(e) (n')est pas que $\mathrm{j}(\mathrm{e})$ (ne) veux pas c'est que $\mathrm{j}(\mathrm{e})(\mathrm{ne})$ peux pas

14. AS : madame $j(e)$ comprends très bien mais moi je (ne) peux pas le reprendre donc je suis obligé de vous le laisser

15. $\mathrm{P}$ : bé non vous (ne) comprenez rien sinon vous feriez pas ça

16. AS : (é)coutez madame vous faites comme vous voulez mais moi je (ne) peux pas le reprendre

17. $P$ : bé j(e) fais comme je veux bé non je (ne) fais pas comme je veux xxx je suis tout le temps emmerdée par des crétins comme vous quoi (1s) alors comment on fait quand c'est comme ça $(2 \mathrm{~s})<\mathrm{j}(\mathrm{e})$ fais quoi avec votre truc là regardez voilà vous voyez c(e) que j'en fais moi de votre truc là > ((elle prend le PV, le froisse et le jette))> clac clac hop c'est fini voilà (il n')y en a plus c'est fini voilà

18. AS : ah c'est c'est très bien madame donc moi je ferai un rapport et puis vous le recevrez quand même <+

19. $\mathrm{P}$ : eh bé ouais c'est très bien faites un rapport $\mathrm{j}(\mathrm{e})(\mathrm{n}$ ') en ai rien à cirer de vos rapports de MERde bon allez foutez-moi là foutez moi la paix quoi

20. AS : allez merci madame au revoir

21. $P$ : ouais c'est ça merci ouais

41 L'automobiliste commence par argumenter (tours 1 à 6), dans une forme d'évidence, sur le fait qu'elle va partir et donc que le PV n'a pas lieu d'être. Or, cet argument n'est pas recevable, non pas en soi, mais parce que le règlement empêche de retirer un $\mathrm{PV}$ qui est terminé. Face à ce fait de droit, elle tente une autre stratégie, la narration, qui va jouer sur un autre registre, la compassion et donc l'appel à la clémence. Le pathos est l'effet émotionnel produit sur l'allocutaire et le recours aux émotions sert autant le polémiste à convaincre son auditoire de ses bons arguments qu'à susciter à son égard une certaine compassion. Si dans les discours politiques, les émotions sont à penser à travers des croyances et des valeurs liées à des jugements d'ordre moral - sentiment d'injustice par exemple - (Charaudeau 2008), dans les relations interpersonnelles, elles 
servent aussi à entretenir l'ethos du polémiste, dans des formes de sympathie, de compassion ou de compréhension qui participent de la prise en compte du sujet.

Le récit de P. (tour 7) s'articule autour d'une tension narrative et donc de personnages papa, maman et l'enfant - d'événements - anniversaire de la petite fille, cadeau à acheter, père qui les a abandonnées, argent qui manque à la mère - et de recours à l'émotion (7. P. j(e) voulais qu'elle (ne) se sente pas : enfin vous voyez $j(e)$ voulais faire un geste quoi). P. interpelle le spectateur (7. P : enfin vous voyez) pour le laisser interpréter, voire s'approprier le récit et participer à la situation pathémique. D'ailleurs, pour ne pas se laisser prendre au jeu, l'agent, désolé, n'a d'autre recours que de faire appel à son impuissance (8. AS : j(e) comprends bien madame mais bon nous une fois qu'on a fini le PV on est obligés de l(e) poser), argument qui va se fossiliser (14. AS : je peux je (ne) peux rien faire madame) parce qu'il n'en a pas d'autres, ce qui le met dans une position peu affable. L'automobiliste s'appuie alors sur l'assertion avancée par l'agent (10. AS : par contre vous avez quarante cinq jours pour le payer) pour la réfuter et créer un effet d'apitoiement. Elle revient, face à une triste situation familiale, au sacrifice qu'elle fait pour sa fille (tour 11). Elle tente ainsi de susciter d'une part de la pitié, émotion reliée à la fois à des valeurs morales (il faut protéger les enfants) et au sentiment d'injustice (Amossy 2010 : 188), et, d'autre part, de l'indignation, qui est « l'effet d'une action dont on peut imputer la responsabilité à un agent » (Micheli 2008 : 136), en accusant le père.

43 Le récit est donc assez élaboré, il a pour fonction d'attirer l'attention de celui qui écoute, de l'amadouer, de le rendre acteur du récit oral et de susciter son émotion pour mieux le rendre à sa cause. La résolution de la tension du récit narratif devrait donc être ici le fait d'enlever le PV mais comme ça ne fonctionne pas (tour 12), qu'elle n'a pas réussi à convaincre son interlocuteur, rompu sans doute à ce genre de rhétorique, elle entre résolument dans l'attaque personnalisée et la condamnation sur le faire et sur l'être. L'automobiliste commence par attaquer le «faire », ce qui est moins violent que "l'être », (13. P : mais allez mais c'est débile votre fonctionnement là (en)fin ça sert à rien c'est con quoi parce que de toutes façons $\mathrm{j}(\mathrm{e})$ peux pas $\mathrm{l}(\mathrm{e})$ payer ((il pose le PV sur le pare-brise)). Mais, très vite, elle attaque sur l'être (15. P : bé non vous comprenez rien sinon vous feriez pas ça) avec un reproche puis une insulte accompagnée de gros mots, seuil de bascule dans la violence verbale fulgurante (17. P : je suis toujours emmerdée par des crétins comme vous). Enfin, elle effectue un passage à l'acte par disqualification et déconsidération de la fonction et du travail accompli (tour 17), elle déchire le PV qu'elle nomme "votre truc" avec mépris (tour 17) et renchérit par une insulte indirecte (19. $\mathrm{P}: \mathrm{j}(\mathrm{e})(\mathrm{n}$ ') en ai rien à cirer de vos rapports de MERde).

\section{Conclusion}

Les argumentations asymétriques prédisposent le locuteur en position basse à ne pas attaquer trop directement le représentant hiérarchique, c'est-à-dire à ne pas rentrer directement ni dans une montée en tension fulgurante ni dans l'insulte. Parce qu'il connaît tout de même les risques (sanction, verbalisation, poursuite, etc.) d'une confrontation directe, il aura tendance, au moins au début de l'échange, à éviter des condamnations du faire ou, pire, de l'être (provocation, menace, insulte par exemple) mais usera de constructions argumentatives élaborées (recours à la justification, à la réfutation, à la concession ou au récit pathémique) dans une stratégie de 
contournement. Parce que les représentants de l'institution refusent d'entendre les arguments émis et en rappelle aux règlements, l'interlocuteur, qui essuie des échecs argumentatifs, soit concède sa faute, soit entre dans un conflit plus ouvert dont il devra assumer les conséquences.

Par ailleurs, on se rend compte que dans les saynètes symétriques (passagers dans un bus, scène de famille, par exemple), les condamnations de l'être directes sont beaucoup plus fréquentes tandis que le recours à l'argumentation fait figure d'exception. Les interlocuteurs entrent alors beaucoup plus rapidement dans la confrontation et dans une montée en tension fulgurante. Quand une relation asymétrique bascule (comme c'est en partie le cas à la fin de l'altercation entre l'ASVP et l'automobiliste) dans une relation symétrique avec force disqualifications voire insultes, les risques encourus sont lourds de conséquences, pour le détenteur de l'autorité qui se sent atteint dans sa fonction voire sa personne et pour l'interlocuteur qui peut être sanctionné (recours à la police notamment).

Ainsi, et pour finir, si ces procédés permettent, au début de l'interaction d'être en confrontation sans trop grande agressivité, leurs échecs dans la négociation font basculer dans une violence verbale fulgurante plus caractérisée.

\section{Conventions de transcription}

\begin{tabular}{|l|l|}
\hline Soulignement & chevauchements \\
\hline+ & micro-pause \\
\hline$(2 \mathrm{~s})$ & pauses en secondes \\
\hline \hline exTRA & segment accentué, majuscules \\
\hline \hline$(($ rire $)) ;(($ clin d'œil $)) ;(($ soulèvesa tasse $)) ;$ & $\begin{array}{l}\text { phénomènes non transcrits, non verbal, } \\
\text { contextualisation italiques }\end{array}$ \\
\hline$<>$ & délimitation des phénomènes entre $(())$ \\
\hline$:$ & allongement vocalique \\
\hline$+>$ & hétéro interruption \\
\hline par- ; il est par- parti & troncation (ratage) \\
\hline$($ ill $)$ y a ; i(l) dit & segment non réalisé \\
\hline xxx & segment incompréhensible \\
\hline
\end{tabular}




\section{BIBLIOGRAPHIE}

Amossy, Ruth. 2010. L'argumentation dans le discours (Paris : Armand Colin)

Amossy, Ruth \& Marcel Burger (éds). 2011. Polémiques médiatiques et journalistiques : le discours polémique en question, Semen 31

Brown, Penelope \& Stephen Levinson. 1987. Politeness. Some universals in language use (Cambridge : Cambridge University Press)

Charaudeau, Patrick. 2008. « Pathos et discours politique », Mickaël Rinn (éd.), Emotions et discours, (Rennes : Presses Universitaires de Rennes), 49-59

Goffman, Erwin. [1973] 2000. La mise en scène de la vie quotidienne (Paris : Minuit)

Laforest, Marty. 2002. «Scenes of family life : complaining in everyday conversation », Journal of Pragmatics 34, 1595-1620

Laforest, Marty \& Claudine Moïse. 2010. « Entre reproche et insulte, comment définir les actes de condamnation ", De la satire à la violence verbale. Quand l'humour-l'humeur engendre l'affrontement, Msh Paris 8, 25 novembre, communication.

Laforest Marty \& Diane Vincent. 2004. «La qualification péjorative dans tous ses états ", Lagorgette, Dominique \& Pierre Larrivée (éds). Les insultes : approches sémantiques et pragmatiques, Langue française $144,59-81$

Laforest Marty \& Diane Vincent (éds). 2006. Les interactions asymétriques (Montréal : Nota bene)

Micheli, Raphaël. 2008. « La construction argumentative des émotions : pitié et indignation dans le débat parlementaire de 1908 sur l'abolition de la peine de mort », Rinn Mickaël (éd.), Emotions et discours (Presses Universitaires de Rennes), 127-141

Moïse, Claudine. 2006. « Analyse de la violence verbale : quelques principes méthodologiques », Actes des xxvieJournées d'étude sur la parole, 12-16 juin, Dinard [en ligne : http://jep2006.irisa.fr/ JEP06_ACTES.PDF, retiré le 10 février 2012]

Moïse, Claudine. 2012. Violence verbale, fulgurances au quotidien, DVD (deux fois trois heures) (Montpellier Languedoc-Roussillon : Crdp)

Moïse, Claudine \& Christina Schultz-Romain. 2010. « Violence verbale et listes de discussions : les argumentations polémiques », Pierozak, Isabelle (éd), Du «terrain » à la relation : expériences de l'internet et questionnements méthodologiques, Cahiers de l'institut de linguistique de Louvain 36/2, 113-133

Perelman, Chaim \& Lucie Olbrechts-Tyteca. [1958], 1988. Traité de $\Gamma$ argumentation, (Bruxelles : Presses de l'Université de Bruxelles)

Perrin, Laurent. 1996. L'ironie mise en trop, du sens des énoncés hyperboliques et ironiques (Paris : Kimé)

Plantin, Christian. 1996. L'argumentation (Paris : Mémo Seuil)

Plantin, Christian, Doury, Marianne \& Véronique Traverso. (éds). 2000. Les émotions dans les interactions (Presses universitaires de Lyon)

Rinn, Mickaël. 2008. « Introduction », Mickaël Rinn (éd.), Emotions et discours, (Presses Universitaires de Rennes), 13-21 
Sacks, Harvey. 1992. Lectures on Conversation, Volumes I and II. Ed. G. Jefferson with Introduction by E.A. Schegloff, Blackwell (Oxford : Blackwell)

Vincent, Diane \& Troy Heisler. 1999. «L'anticipation d'objections : prolepse, concession et réfutation dans la langue spontanée », Revue québécoise de linguistique appliquée, 27 : 1, 15-31

\section{NOTES}

1. Merci à Carole Gayet, à Marty Laforest, Christina Schultz Romain et à Diane Vincent.

\section{RÉSUMÉS}

Si nous avons défini la violence verbale fulgurante comme une montée en tension caractérisée par des actes menaçants directs (provocation, menace, insultes...) et la violence polémique comme un discours à visée argumentative mobilisant des procédés discursifs indirects (implicites, ironie...), on ne peut considérer ces deux types de discours comme hermétiques. À travers des scènes de violences verbales quotidiennes dans l'espace public et institutionnel (contrôles, convocations, verbalisations...), constituées pour un DVD pédagogique, il s'agit de montrer comment dans des interactions caractérisées par la violence fulgurante, certains procédés argumentatifs particuliers et que nous décrirons, sont utilisés, avec force efficacité, à des fins de déstabilisation et de prise de pouvoir sur l'autre.

Our research has defined severe verbal abuse as built up tension characterized by directly threatening acts (such as provocation, threats, insults), and polemical violence as argumentative discourse which mobilizes indirect discursive devices, such as implicit discourse relations and irony. Yet, neither type of discourse can be considered to be impervious to mutual influence. Based on the content of an educational DVD featuring acted out scenes of daily verbal abuse taking place in public and institutional spaces (i.e., checks, summons, fines), we will show how specific argumentative devices, which we will describe, are very efficiently used within interactions that are characterised by severe abuse, with the aim of destabilizing and taking control over somebody.

\section{INDEX}

Mots-clés : concession, ironie, lien causal, mise en récit, montée en tension, pathos, violence verbale

Keywords : built up tension, compromise, irony, pathos, storytelling, verbal abuse

\section{AUTEUR}

\section{CLAUDINE MOÏSE}

Université Stendhal, Grenoble 3 